\title{
DOWKER-TYPE THEOREMS FOR HYPERCONVEX DISCS
}

\author{
G. FEJES TÓTH AND F. FODOR
}

\begin{abstract}
A hyperconvex disc of radius $r$ is a planar set with nonempty interior that is the intersection of closed circular discs of radius $r$. A convex disc-polygon of radius $r$ is a set with nonempty interior that is the intersection of a finite number of closed circular discs of radius $r$. We prove that the maximum area and perimeter of convex disc- $n$-gons of radius $r$ contained in a hyperconvex disc of radius $r$ are concave functions of $n$, and the minimum area and perimeter of disc- $n$-gons of radius $r$ containing a hyperconvex disc of radius $r$ are convex functions of $n$. We also consider hyperbolic and spherical versions of these statements.
\end{abstract}

\section{INTRODUCTION AND RESULTS}

Let $K$ denote a convex disc, that is, a compact convex set with non-empty interior in the Euclidean plane $\mathbb{E}^{2}$. Confirming a conjecture of Kershner, Dowker [9] proved that the maximum area of $n$-gons inscribed in $K$ is a concave function of $n$, while the minimum area of $n$-gons circumscribed about $K$ is a convex function of $n$. Dowker observed that the argument of his proof also shows that if $K$ is centrally symmetric, then among the $2 n$-gons of maximum area inscribed in $K$, as well as among the $2 n$-gons of minimum area circumscribed about $K$, there is one that is centrally symmetric with the same centre as $K$.

Dowker's theorems play an important role in the theory of packing and covering in the Euclidean plane. For example, they are essential to L. Fejes Tóth's proof about the packing density of convex discs in a convex hexagon, cf. [12]. L. Fejes Tóth [13], Molnár [20], and Eggleston [10] observed independently of each other that Dowker's results remain true if the word "area" is replaced by "perimeter". The results about centrally symmetric discs was generalized in [11] for $k n$-gons inscribed in and circumscribed about a convex disc with $k$-fold rotational symmetry.

Convex sets in the plane are intersections of half-planes. As a natural strengthening of convexity we study sets that are the intersection of discs of radius $r$. Properties of such sets were studied in several papers and different authors used different names for them. It appears that it was Mayer [19] who first investigated such sets. He studied them in the more general setting of Minkowski geometry and called them "überkonvex". He characterized such sets with the property that together with any two points of them the shorter arcs of both circles of radius $r$ connecting the two points belong to the set. Mayer's paper inspired further research in the 1930s and the 1940s (cf. for example Blanc [5], Buter [6], Pasqualini [21], Santaló [22], van der Corput [23], Vincensini [24], see also the survey paper by Danzer, Grünbaum, and Klee [8]), which, however, was forgotten by the turn of the century. More than seven decades after Mayer, Bezdek et al. [4] and Kupitz et al. $[17,18]$ made a thorough investigation of such sets in $\mathbb{E}^{n}$ (for more information see

Date: February 10, 2014. 
also [1] and [2]). Bezdek et al. [4] and Kupitz et al. [17,18] call such sets spindle convex, while L. Fejes Tóth $[15,16]$, who proved packing and covering theorems for them, used the term $r$-convex. We use the English translation of Mayer's phrase. A planar set is hyperconvex with radius $r$ if it is the intersection of circular discs of radius $r$. Note that if we consider half-planes as circles of infinite radius, then we obtain the family of linearly convex sets for $r=\infty$. A hyperconvex disc of radius $r$ is a compact hyperconvex set with radius $r$ and with nonempty interior. A disc-polygon of radius $r<\infty$ is the intersection of a finite number of discs of radius $r$ in such a way that the interior of the set is nonempty. We shall assume that whenever a disc-polygon is represented as the intersection of some discs, each disc is essential, that is, if discarded, then the intersection changes. Thus, the boundary of a disc-polygon of radius $r$ consists of a finite number of radius $r$ circular arcs of positive length, called sides, each of which is part of the boundary of a unique generating disc. The sides follow in a natural cyclic order on the boundary of the disc-polygon. Two consecutive sides in this order meet in a vertex, except in the case of disc-2-gons in which two consecutive sides intersect in a pair of vertices. Therefore, if a disc-polygon of radius $r$ is the intersection of $n>1$ essential discs, then it has exactly $n$ sides and $n$ vertices. We will call such sets disc-n-gons of radius $r$.

In this article we extend Dowker's theorem for the case when $K$ is a hyperconvex disc of radius $r$ and the approximating objects are disc-polygons of radius $r$. Since the value of $r$ is fixed throughout the article, we suppress its notation unless this omission may cause confusion.

Let $a_{i}(n)$ and $p_{i}(n)$ denote the maximum area and maximum perimeter of convex disc-polygons with at most $n$ vertices contained in $K$. Let $a_{c}(n)$ and $p_{c}(n)$ denote the minimum area and minimum perimeter of convex disc-polygons with at most $n$ vertices containing $K$. It is easily seen that $a_{i}(n)$ and $p_{i}(n)$ are realized by disc-polygons that are inscribed in $K$ in the sense that their vertices are on the boundary of $K$. Similarly, $a_{c}(n)$ and $p_{c}(n)$ are realized by disc-polygons that are circumscribed about $K$ meaning that their sides are tangential to the boundary of $K$.

Theorem 1. We have, for $n \geq 4$,

i) $a_{c}(n-1)+a_{c}(n+1) \geq 2 a_{c}(n)$,

ii) $p_{c}(n-1)+p_{c}(n+1) \geq 2 p_{c}(n)$,

iii) $a_{i}(n-1)+a_{i}(n+1) \leq 2 a_{i}(n)$,

iv) $p_{i}(n-1)+p_{i}(n+1) \leq 2 p_{i}(n)$.

Theorem 2. If $K$ has $k$-fold rotational symmetry, then there are disc-polygons $P_{c a}$ and $P_{c p}$ with at most $k n$ vertices circumscribed about $K$, as well as disc-polygons $P_{i a}$ and $P_{i p}$ with at most $k n$ vertices inscribed in $K$ such that all of them have $k$-fold rotational symmetry with the same centre as $K$ and area $\left(P_{c a}\right)=a_{c}(k n)$, $\operatorname{per}\left(P_{c p}\right)=p_{c}(k n)$, area $\left(P_{i a}\right)=a_{i}(k n)$, and $\operatorname{per}\left(P_{i p}\right)=p_{i}(k n)$.

We note that the above theorems were proved by Bezdek et al. in [4] for the special case when $K$ is a closed circular disc of radius $r<1$. We stated Theorem 1 for $n \geq 4$, so that it includes the case $r=\infty$, as well. If $r<\infty$, then also digons can occur and Theorem 1 holds for $n \geq 3$. 


\section{ProOfS}

Since the case $r=\infty$ of our theorems is well-known, we shall restrict our attention to the case when $r$ is finite. We will use circle-polygons of radius $r$. Following Bezdek et al. (cf. [4], page 224) we define such circle-polygons as described below. Let $v_{1}, \ldots, v_{n}$ be a sequence of points such that $d\left(v_{i}, v_{i+1}\right) \leq 2 r$ for $i=1, \ldots, n$ and with the convention that $v_{n+1}=v_{1}$. Let $v_{i} v_{i+1}$ denote one of the shorter circular arcs of radius $r$ incident with $v_{i}$ and $v_{i+1}$. The union of the $\operatorname{arcs} v_{1} v_{2}, \ldots, v_{n} v_{1}$ is called a circle-polygon of radius $r$, which we denote by $C$. We call the points $v_{i}$ the vertices and the circular arcs $v_{i} v_{i+1}$ the sides of $C$. Note that a circle-polygon of radius $r$ does not necessarily bound a convex domain and it may be self-intersecting. The boundary of a convex disc-polygon of radius $r$ is an example of circle-polygons of radius $r$. Since $r$ is fixed, henceforth we omit it from our notation.

We fix an orientation of the plane which induces a natural orientation of any circular arc. Thus, a circular arc has a well-defined initial and terminal point. We say that a circle-polygon is orientable (or proper) if the induced orientation of its sides determines an orientation of the entire circle-polygon. Disc-polygons are obviously orientable. In this paper we only consider orientable circle-polygons. Subsequently, we will omit the word "orientable".

Let $P=\left\{C_{1}, \ldots, C_{n}\right\}$ be a finite collection of closed circle-polygons. We call such a collection a multiple circle-polygon. It encloses a signed multiset in which the multiplicity of a point $p$ is defined as $\sum_{i=1}^{n} \chi_{i}(p)$, where $\chi_{i}(p)$ is the winding number of $C_{i}$ around $p$ while going around $C_{i}$ in the positive direction. The area and the perimeter of $P$ are defined as

$$
\operatorname{area}(P)=\sum_{k \in \mathbb{Z}} k \cdot \operatorname{area}(\{p: \chi(p)=k\}),
$$

and

$$
\operatorname{per}(P)=\sum_{i=1}^{n} \operatorname{per}\left(C_{i}\right)
$$

respectively. The intersection of the discs corresponding to the sides of the circlepolygons $C_{1}, \ldots, C_{n}$ is the core of $P$. In the proofs of Theorem 1 and 2 double circle-polygons play a crucial role. These are those multiple circle-polygons for which the core is not empty and the points of the core have multiplicity 2.

Following Bezdek, Csikós, and Connelly (cf. [3], p. 55) and Bezdek et al. (cf. [4], p. 203), we introduce the arc-distance of radius $r$ of two points. More precisely, let $x, y \in \mathbb{E}^{2}$ such that $d(x, y) \leq 2 r$. Then their arc-distance of radius $r$, denoted by $\varrho_{r}(x, y)$, is defined as the length of the shorter circular arc of radius $r$ connecting $x$ and $y$. It is noted in [3] that the arc-distance is not a metric. In fact, its behaviour is described in the following two statements from [3], which we cite in slightly modified forms.

Proposition 1 (Bezdek, Csikós, and Connelly [3], Lemma 1). Let $r>0$ and $x, y, z \in \mathbb{E}^{2}$ such that $d(x, y) \leq 2 r, d(x, z) \leq 2 r$, and $d(y, z) \leq 2 r$. Let I denote the intersection of the two discs of radius $r$ whose boundary contains $x$ and $z$. Then $\varrho_{r}(x, y)+\varrho_{r}(y, z)>\varrho_{r}(x, z), \varrho_{r}(x, y)+\varrho_{r}(y, z)=\varrho_{r}(x, z)$ or $\varrho_{r}(x, y)+\varrho_{r}(y, z)<$ $\varrho_{r}(x, z)$ according as $y \notin I, y \in \operatorname{bd} I$ or $y \in \operatorname{int} I$.

Proposition 2 (Bezdek, Csikós, and Connelly [3], Lemma 2). Let $r>0$ and $x, y, z, w \in \mathbb{E}^{2}$ be the vertices of a disc-quadrilateral of radius $r$, listed in a cyclic 
order. Then

$$
\varrho_{r}(x, y)+\varrho_{r}(z, w)<\varrho_{r}(x, z)+\varrho_{r}(y, w),
$$

that is, the sum of the arc-lengths of the diagonals is larger than the sum of the arc-lengths of any two nonadjacent sides.

We begin with the proofs of i) and ii) of Theorem 1 and the corresponding cases of Theorem 2.

Let $K$ be a hyperconvex disc and $P$ a double circle-polygon circumscribed about $K$. Let $s_{1}, \ldots, s_{n}$ be the sides of $P$. We choose the notation so that $s_{i-1}$ and $s_{i}$ are neighbors meeting in a vertex $v_{i}$. If $P$ is a single circle-polygon winding twice around the core of $P$, this can be achieved by the convention $s_{n}=s_{0}$ and $s_{n+1}=s_{1}$. On the other hand, if $P$ is the union of two circle-polygons, with $l$ and $m$ sides, say, then we label the sides of the $l$-gon by $s_{1}=s_{l+1}, s_{2}, \ldots, s_{l}=s_{0}$ and the sides of the $m$-gon by $s_{l+3}=s_{l+m+3}, s_{l+4}, \ldots, s_{l+m+2}=s_{l+2}$. Let $C_{i}$ denote the circle containing the side $s_{i}, o_{i}$ its centre and $D_{i}$ the discs bounded by $C_{i}$. To each side of $P$ we assign a point $t_{i} \in s_{i} \cap \mathrm{bd} K$. If $s_{i} \cap \mathrm{bd} K$ is not a single point, then we can choose $t_{i}$ arbitrarily with the restriction that if $C_{i}=C_{j}$ then $t_{i}=t_{j}$.

We note that to any permutation of the points $t_{i}$ corresponds a double circlepolygon in which the side associated with $t_{i}$ is the $\operatorname{arc}$ of $C_{i}$ lying in the intersection of the discs associated with the points preceding and following $t_{i}$ in the permutation. Arrange the points $t_{i}$ in the order as they follow on bd $K$ while going around in the positive direction. As there might be coincident points among the $t_{i}$ 's, we have to specify the order of such points. If $t_{i}=t_{j}$ and $C_{i}=C_{j}$, then the order of $t_{i}$ and $t_{j}$ can be chosen arbitrarily. Suppose that $t_{i}=t_{j}$ but $C_{i} \neq C_{j}$. If the arc $v_{i-1} t_{i}$ is contained in $D_{j}$ then $t_{i}$ precedes $t_{j}$, and if the arc $v_{j-1} t_{j}$ is contained in $D_{i}$ then $t_{j}$ precedes $t_{i}$.

Enumerate the points $t_{i}$ by taking every second from the above natural order and let $P^{*}$ be the double circle-polygon corresponding to this order. The statements of our theorems for circumscribed disc polygons are immediate corollaries of the following

Lemma 1. We have

$$
\text { area }(P) \geq \operatorname{area}\left(P^{*}\right) \quad \text { and } \quad \operatorname{per}(P) \geq \operatorname{per}\left(P^{*}\right) .
$$

Note that if $K$ is a disc-polygon with at most $n$ sides, then inequalities i) and ii) of Theorem 1 are obvious. Thus, we may assume that $K$ is not a disc-polygon with at most $n$ sides. Then there are disc-l-gons circumscribed about $K$ for all $l \leq n+1$. Now, if $P$ is the union of two simple circle-polygons $Q$ and $R$ circumscribed about $K$ with $n-1$ and $n+1$ sides, respectively, then $P^{*}$ consists of two simple circlepolygons $Q^{\prime}$ and $R^{\prime}$ with $n$ sides. Thus

$$
\begin{aligned}
\operatorname{area}(Q)+\operatorname{area}(R)=\operatorname{area}(P) \geq \operatorname{area}\left(P^{*}\right)=\operatorname{area}\left(Q^{\prime}\right)+\operatorname{area}\left(R^{\prime}\right) \\
\geq 2 \min \left\{\text { area }\left(Q^{\prime}\right), \text { area }\left(R^{\prime}\right)\right\},
\end{aligned}
$$

and

$$
\begin{aligned}
\operatorname{per}(Q)+\operatorname{per}(R)=\operatorname{per}(P) \geq \operatorname{per}\left(P^{*}\right)=\operatorname{per}\left(Q^{\prime}\right)+ & \operatorname{per}\left(R^{\prime}\right) \\
& \geq 2 \min \left\{\operatorname{per}\left(Q^{\prime}\right), \operatorname{per}\left(R^{\prime}\right)\right\} .
\end{aligned}
$$

Suppose now that $K$ has $k$-fold rotational symmetry. Again, we may assume that $K$ is not a disc-polygon with at most $k n$ vertices, as otherwise the statement of 
Theorem 2 is obvious. Consider a disc-polygon $Q_{0}$ with $n k$ vertices circumscribed about $K$. The rotations through $2 \pi / j, j=1, \ldots, k-1$ carrying $K$ onto itself transform $Q_{0}$ into $Q_{1}, \ldots, Q_{k-1}$. As above, we associate with each side of these disc-polygons a point of tangency with bd $K$. Any pair $Q_{i}, Q_{j}, 0 \leq i<j \leq k-1$, forms a double circle-polygon $P$. If $P$ does not coincide with the corresponding $P^{*}$, then we replace $Q_{i}$ and $Q_{j}$ by the two disc-polygons forming $P^{*}$. Obviously, the new polygons have $n k$ vertices. By this replacement neither the total area nor the total perimeter of the disc-polygons $Q_{0}, \ldots, Q_{k-1}$ increases. We repeat this process until all double circle-polygons $P$ formed by a pair $Q_{i}, Q_{j}$ coincide with the corresponding $P^{*}$.

Let $T$ be the union of the selected points of tangency of all sides of $Q_{0}, \ldots, Q_{k-1}$. Let us order the elements of $T$ cyclically on bd $K$. T has $k^{2} n$ elements, and by construction, it has $k$-fold rotational symmetry. Therefore a rotation around the centre of $K$ by an angle of $2 \pi / k$ carries each element of $T$ in the $k n$-th element following it in the cyclic order. The disc-polygons resulting from the above procedure have the property that between any two tangency points of consecutive sides there are exactly $k-1$ tangency points of sides of other $Q_{i}$ 's. Thus any of these disc-polygons arises by taking the intersection of the discs corresponding to every $k$-th tangency point. Therefore all of them have $k$-fold rotational symmetry.

Proof of Lemma 1. With each vertex $v_{i}$ of $P$ we associate the arc $a_{i}=\widehat{t_{i-1} t_{i}}$ of bd $K$ between $t_{i-1}$ and $t_{i}$. If $P=P^{*}$, then the statement of Lemma 1 is obvious. If $P \neq P^{*}$, then for some $i$ and $j$ we have $a_{j} \subset a_{i}$. Let $C_{j-1}$ intersect $s_{i}$ in $v_{j}^{\prime}$ and let $C_{j}$ intersect $s_{i-1}$ in $v_{i}^{\prime}$. We obtain a new double circlepolygon $P^{\prime}$ by replacing the sides $s_{i-1}, s_{i}, s_{j-1}$, and $s_{j}$ by the $\operatorname{arcs} v_{i-1} v_{i}^{\prime}, v_{j}^{\prime} v_{i+1}$, $v_{j-1} v_{j}^{\prime}$, and $v_{i}^{\prime} v_{j+1}$, respectively, so that the cyclic order of the vertices of $P^{\prime}$ is $\ldots v_{i-2} v_{i-1} v_{i}^{\prime} v_{j+1} \ldots v_{j-1} v_{j}^{\prime} v_{i+1} v_{i+2} \ldots v_{i-2}$. Let $t$ denote the area of the region $R$ enclosed by the $\operatorname{arcs} v_{i} v_{j}^{\prime}, v_{j}^{\prime} v_{j}, v_{j} v_{i}^{\prime}$, and $v_{i}^{\prime} v_{i}$, where we allow that $R$ is degenerate and its area is zero (see Figure 1).

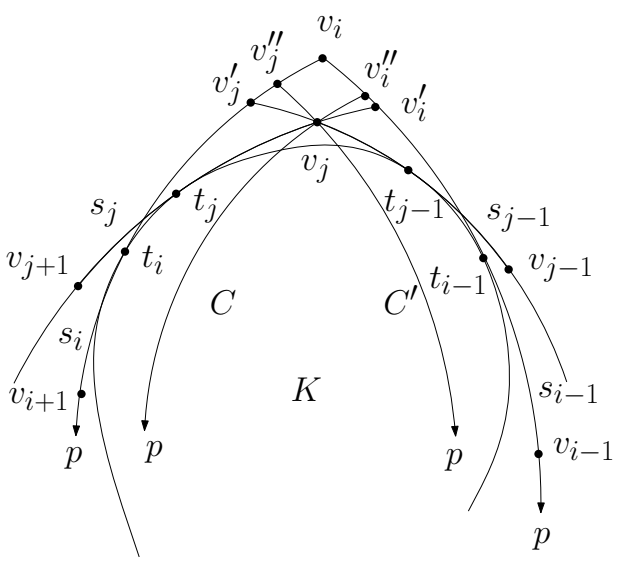

FIGURE 1.

Then

$$
\text { area }(P)=\operatorname{area}\left(P^{\prime}\right)+t \geq \operatorname{area}\left(P^{\prime}\right)
$$


We show that also

$$
\operatorname{per}(P) \geq \operatorname{per}\left(P^{\prime}\right)
$$

We have

$$
\operatorname{per}\left(P^{\prime}\right)=\operatorname{per}(P)+\varrho_{r}\left(v_{j}^{\prime}, v_{j}\right)+\varrho_{r}\left(v_{j}, v_{i}^{\prime}\right)-\varrho_{r}\left(v_{i}^{\prime}, v_{i}\right)-\varrho_{r}\left(v_{i}, v_{j}^{\prime}\right),
$$

thus we have to show that

$$
\varrho_{r}\left(v_{i}^{\prime}, v_{i}\right)+\varrho_{r}\left(v_{i}, v_{j}^{\prime}\right) \geq \varrho_{r}\left(v_{j}^{\prime}, v_{j}\right)+\varrho_{r}\left(v_{j}, v_{i}^{\prime}\right) .
$$

Let $p$ be the point of intersection of $C_{i-1}$ and $C_{i}$ different from $v_{i}$. Let $C$ and $C^{\prime}$ be the circles of radius $r$ passing through the points $v_{j}$ and $p$. Denote their centres with $o$ and $o^{\prime}$, respectively. Since $p \in D_{j-1} \cap D_{j}$, one of these circles, say $C$ intersects the $\operatorname{arc} v_{i}^{\prime} v_{i}$, while $C^{\prime}$ intersects the $\operatorname{arc} v_{i} v_{j}^{\prime}$. Write $v_{i}^{\prime \prime}=C \cap v_{i}^{\prime} v_{i}$ and $v_{j}^{\prime \prime}=C^{\prime} \cap v_{i} v_{j}^{\prime}$.

Observe that $\measuredangle p o_{i} v_{i}=\pi-\measuredangle o_{i-1} p o_{i}, \measuredangle p o_{i} v_{j}^{\prime \prime}=\pi-\measuredangle o_{i} p o^{\prime}, \measuredangle p o v_{i}^{\prime \prime}=\pi-\measuredangle o_{i-1} p o$, and $\measuredangle p o v_{j}=\pi-\measuredangle o p o^{\prime}$. Hence

$$
\measuredangle v_{i} o_{i} v_{j}^{\prime \prime}=\measuredangle p o_{i} v_{i}-\measuredangle p o_{i} v_{j}^{\prime \prime}=\measuredangle o_{i} p o^{\prime}-\measuredangle o_{i-1} p o_{i}=\measuredangle o_{i-1} p o^{\prime}
$$

and

$$
\measuredangle v_{i}^{\prime \prime} o v_{j}=\measuredangle p o v_{i}^{\prime \prime}-\measuredangle p o v_{j}=\measuredangle o^{\prime} p o-\measuredangle o_{i-1} p o=\measuredangle o_{i-1} p o^{\prime} .
$$

It follows that $\varrho_{r}\left(v_{i}, v_{j}^{\prime \prime}\right)=\varrho_{r}\left(v_{i}^{\prime \prime}, v_{j}\right)$. In the same way we see that $\varrho_{r}\left(v_{i}, v_{i}^{\prime \prime}\right)=$ $\varrho_{r}\left(v_{j}^{\prime \prime}, v_{j}\right)$.

Obviously, $v_{i}^{\prime \prime}$ is not contained in the interior of the intersection of the two circles of radius $r$ passing through $v_{i}$ and $v_{i}^{\prime}$. It follows by Proposition 1 that $\varrho_{r}\left(v_{j}, v_{i}^{\prime}\right) \leq \varrho_{r}\left(v_{j}, v_{i}^{\prime \prime}\right)+\varrho_{r}\left(v_{i}^{\prime}, v_{i}^{\prime \prime}\right)$. Similarly we have $\varrho_{r}\left(v_{j}, v_{j}^{\prime}\right) \leq \varrho_{r}\left(v_{j}, v_{j}^{\prime \prime}\right)+$ $\varrho_{r}\left(v_{j}^{\prime}, v_{j}^{\prime \prime}\right)$. Therefore

$$
\begin{aligned}
\varrho_{r}\left(v_{i}^{\prime}, v_{i}\right)+\varrho_{r}\left(v_{i}, v_{j}^{\prime}\right) & =\varrho_{r}\left(v_{i}^{\prime}, v_{i}^{\prime \prime}\right)+\varrho_{r}\left(v_{i}^{\prime \prime}, v_{i}\right)+\varrho_{r}\left(v_{j}^{\prime}, v_{j}^{\prime \prime}\right)+\varrho_{r}\left(v_{j}^{\prime \prime}, v_{i}\right) \\
& =\varrho_{r}\left(v_{i}^{\prime}, v_{i}^{\prime \prime}\right)+\varrho_{r}\left(v_{j}, v_{i}^{\prime \prime}\right)+\varrho_{r}\left(v_{j}^{\prime}, v_{j}^{\prime \prime}\right)+\varrho_{r}\left(v_{j}, v_{j}^{\prime \prime}\right) \\
& \geq \varrho_{r}\left(v_{j}^{\prime}, v_{j}\right)+\varrho_{r}\left(v_{j}, v_{i}^{\prime}\right),
\end{aligned}
$$

as claimed.

We obtain a sequence of double circle-polygons with non-increasing areas and perimeters by iterating this construction until there are no vertices with the property that the arc of bd $K$ assigned to one contains the arc assigned to the other. Thus the result of this process is $P^{*}$.

Now we turn to the proof of the statements of our theorems dealing with inscribed disc-polygons. Let $P$ be a double circle-polygon inscribed in $K$ with sides $s_{1}, \ldots, s_{n}$. Again, we assume that $s_{i-1}$ and $s_{i}$ are neighbours meeting in the vertex $v_{i}$ and we index the sides of $P$ in the same way as described before Lemma 1. Our goal is to find a new double circle-polygon inscribed in $K$ whose area and perimeter is not less than that of $P$. We do not consider cases when the area and perimeter of $P$ can be increased in an obvious way. We assume that the vertices of $P$ are not all on an open arc of the boundary of $K$ lying between points of tangency of two parallel supporting lines.

Arrange the vertices of $P$ in the order as they follow on the boundary of $K$. If two vertices coincide, then we may order them arbitrarily. Observe that for two points that are second neighbours in this cyclic order one of the circles passing through the two points contains the core of $P$. By taking every second vertex and 
connecting them by the shorter arc of the respective circle we obtain a new double circle-polygon $P^{*}$ inscribed in $K$.

Lemma 2. We have

$$
\text { area }(P) \leq \operatorname{area}\left(P^{*}\right) \text { and } \operatorname{per}(P) \leq \operatorname{per}\left(P^{*}\right) .
$$

From Lemma 2 the statements of our theorems for the inscribed case follow by essentially the same arguments as above. There is one important difference. The union of two simple circle-polygons circumscribed about $K$ is always a double circlepolygon. For inscribed circle-polygons this is not the case. In order to ensure that we get a double circle-polygon we have to start with circle-polygons which have maximum area or maximum perimeter for the given number of sides. Then the vertices of such a circle-polygon cannot all be on an open arc of the boundary of $K$ lying between points of tangency of two parallel supporting lines. This guarantees that the core of the multiple circle-polygon formed by two such circle-polygons is not empty.

Proof of Lemma 2. With each side $s_{i}=v_{i-1} v_{i}$ of $P$, we associate the arc $a_{i}=$ $\widehat{v_{i-1} v_{i}}$ of bd $K$ between $v_{i-1}$ and $v_{i}$. If $P=P^{*}$, then the statement of Lemma 2 is obvious. If $P \neq P^{*}$, then $a_{j} \subset a_{i}$ for some $i$ and $j$. Let $P^{\prime}$ denote the double circle-polygon inscribed in $K$ whose vertices, listed in positive cyclic order, are the following:

$$
\ldots v_{j-2} v_{j-1} v_{i} v_{i+1} \ldots v_{i-1} v_{j} v_{j+1} \ldots
$$

Applying Proposition 2 to the circle-quadrangle $v_{i-1} v_{j-1} v_{j} v_{i}$ (which may degenerate into a circle-triangle), we obtain that

$$
\operatorname{per}\left(P^{\prime}\right) \geq \operatorname{per}(P) \text {. }
$$

We need to show that

$$
\operatorname{area}\left(P^{\prime}\right) \geq \operatorname{area}(P)
$$

as well.

We begin the proof of this inequality with a technical statement. Consider three parallel lines $L_{1}, L_{2}$ and $M$ such that the distance between $L_{1}$ and $L_{2}$ is less than $2 r$ and $M$ is at equal distance from $L_{1}$ and $L_{2}$. Let $C$ be a circle of radius $r$ intersecting both $L_{1}$ and $L_{2}$. We choose a coordinate system with origin in $M$ so that the points of intersection of $L_{1}$ and $C$ are $u=\left(-x_{0},-y_{0}\right)$ and $v=\left(x_{0},-y_{0}\right)$. For a point $w=\left(x, y_{0}\right)$ inside $C$ we define the region $T=T(x)$ bounded by the circular arcs of radius $r$ connecting the points $u$ and $v, v$ and $w$ and $w$ and $u$, respectively, so that the arcs between $u$ and $v$ and the arc between $v$ and $w$ are outside the triangle $\Delta=u v w$, while the arc between $u$ and $w$ is on the same side of the line $u w$ as $\Delta$ (see Figure 2).

We are going to show that

$$
\text { area }(T(x)) \text { is a monotonically decreasing function of } x \text {. }
$$

Let $S(u, v), S(v, w)$ and $S(w, v)$ denote the circular segments determined by $T(x)$, respectively, as shown on Figure 2. Then

$$
\text { area }(T(x))=\operatorname{area}(\Delta)+\operatorname{area}(S(u, v))+\operatorname{area}(S(v, w))-\operatorname{area}(S(u, w)) \text {. }
$$

Clearly, area $(\Delta)$ and area $(S(u, v))$ are independent of $x$, so we have to show the monotonicity of area $(S(v, w))$ - area $(S(u, w))$. This is obvious in the case when 


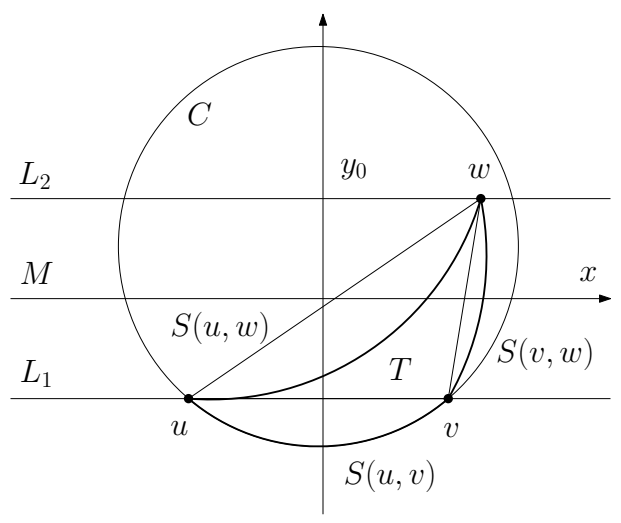

FiguRE 2.

$x \in\left[-x_{0}, x_{0}\right]$ since in this interval area $(S(u, w))$ is monotonically increasing and area $(S(v, w))$ is monotonically decreasing. Observe that

$$
\text { area }(T(x))+\text { area }(T(-x))=2 \text { area }(\Delta)+2 \text { area }(S(u, v))=\text { constant. }
$$

Thus we may assume that $x \geq x_{0}$.

Let $s(l)$ denote the area of a circular segment cut off from a circle of radius $r$ by a chord of length $l$. Further define the function $l(z)$ for $z \geq 0$ as the distance from the point $\left(x,-y_{0}\right)$ to $\left(x+z, y_{0}\right)$. It is easily seen that both $s(l)$ and $l(z)$ are monotonically increasing convex functions. It follows that the compound function $f(z)=s(l(z))$ is also monotonically increasing and convex on its domain. We have

$$
\text { area }(S(v, w)) \text { - area }(S(u, w))=f\left(x+x_{0}\right)-f\left(x-x_{0}\right) .
$$

Now the statement in $(*)$ follows by observing that the right hand side increases by the convexity of $f$.

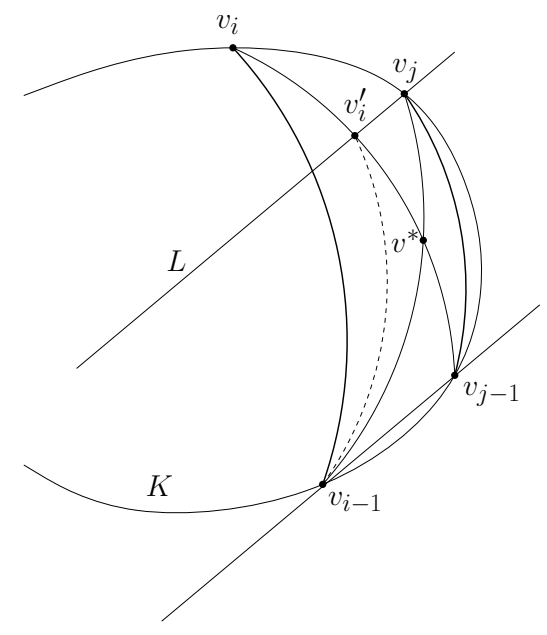

FIGURE 3.

Let $v^{*}$ be the intersection point of the circular $\operatorname{arcs} v_{j-1} v_{i}$ and $v_{i-1} v_{j}$ as shown on Figure 3. In order to prove the inequality area $\left(P^{\prime}\right) \geq$ area $(P)$ we have to 
show that the area of the region bounded by the $\operatorname{arcs} v_{i} v_{i-1}, v_{i-1} v^{*}$ and $v^{*} v_{i}$ is not smaller than the area of the region bounded by the $\operatorname{arcs} v_{j} v_{j-1}, v_{j-1} v^{*}$ and $v^{*} v_{j}$. Equivalently, we can compare the area of the region $R_{1}$ bounded by the arcs $v_{i-1} v_{j-1}, v_{j-1} v_{i}$ and $v_{i} v_{i-1}$ to that of $R_{2}$ bounded by the $\operatorname{arcs} v_{i-1} v_{j-1}, v_{j-1} v_{j}$ and $v_{j} v_{i-1}$. Let $L$ be the line through $v_{j}$ that is parallel to the line incident with $v_{i-1}$ and $v_{j-1}$. It follows by the assumption that the vertices of $P$ are not all on an open arc of the boundary of $K$ lying between points of tangency of two parallel supporting lines that $L$ intersects the circular $\operatorname{arc} v_{j-1} v_{i}$ in a point $v_{i}^{\prime}$. Let $R_{1}^{\prime}$ denote the region bounded by the $\operatorname{arcs} v_{i-1} v_{j-1}, v_{j-1} v_{i}^{\prime}$ and $v_{i}^{\prime} v_{i-1}$. It is clear that area $\left(R_{1}\right) \geq$ area $\left(R_{1}^{\prime}\right)$, and by the auxiliary statement proved above area $\left(R_{1}^{\prime}\right) \geq$ area $\left(R_{2}\right)$.

We obtain a sequence of double circle-polygons with non-decreasing areas and perimeters by iterating this construction until there are no sides with the property that the arc of the boundary of $K$ assigned to one contains the arc assigned to the other. Thus, this process results in $P^{*}$ in a finite number of steps.

\section{Concluding Remarks}

The theorems of Dowker and their extensions for the perimeter hold also on the sphere and in the hyperbolic plane. This was shown by Molnár [20] with the exception of the case concerning the perimeter of circumscribed polygons on the sphere. This last case was settled by L. Fejes Tóth [14] who observed that on the sphere the statements for the perimeter of circumscribed polygons and for the area of inscribed polygons are equivalent by spherical polarity.

With the exception of the case concerning the perimeter of circumscribed discpolygons on the sphere the proofs of our theorems can be carried over to the sphere (for $r \leq \pi / 2$ ) and the hyperbolic plane. The arguments deducing the theorems from Lemma 1 and Lemma 2 do not use the special structure of the geometry. The proofs of the case of area in Lemma 1 and the case of perimeter in Lemma 2 carry over to the hyperbolic plane and the sphere without change. We outline the changes needed in the proofs of the case of perimeter of Lemma 1 in the hyperbolic plane and the case of area of Lemma 2 in the hyperbolic plane and on the sphere.

Proof of the case of perimeter in Lemma 1 in the hyperbolic plane. In this argument we use the same notations as on Figure 1. On the hyperbolic plane we have $\measuredangle p o_{i} v_{i}=2 \operatorname{arccot} \cosh r \tan \left(\frac{1}{2} \measuredangle o_{i-1} p o_{i}\right), \measuredangle p o_{i} v_{j}^{\prime \prime}=2 \operatorname{arccot} \cosh r \tan \left(\frac{1}{2} \measuredangle o_{i} p o^{\prime}\right)$, $\measuredangle p o v_{i}^{\prime \prime}=2 \operatorname{arccot} \cosh r \tan \left(\frac{1}{2} \measuredangle o_{i-1} p o\right)$, and $\measuredangle p o v_{j}=2 \operatorname{arccot} \cosh r \tan \left(\frac{1}{2} \measuredangle o p o^{\prime}\right)$. Hence

$$
\begin{aligned}
\measuredangle v_{i} o_{i} v_{j}^{\prime \prime} & =\measuredangle p o_{i} v_{i}-\measuredangle p o_{i} v_{j}^{\prime \prime} \\
& =2 \operatorname{arccot} \cosh r \tan \left(\measuredangle o_{i-1} p o_{i} / 2\right)-2 \operatorname{arccot} \cosh r \tan \left(\measuredangle o_{i} p o^{\prime} / 2\right)
\end{aligned}
$$

and

$$
\begin{aligned}
\measuredangle v_{i}^{\prime \prime} o v_{j} & =\measuredangle p o v_{i}^{\prime \prime}-\measuredangle p o v_{j} \\
& =2 \operatorname{arccot} \cosh r \tan \left(\measuredangle o_{i-1} p o / 2\right)-2 \operatorname{arccot} \cosh r \tan \left(\measuredangle o p o^{\prime} / 2\right) .
\end{aligned}
$$

It is easily seen that the function $y=\operatorname{arccot}(\cosh r \tan x)$ is increasing and convex. We have

$$
\measuredangle o_{i-1} p o_{i} / 2-\measuredangle o_{i} p o^{\prime} / 2=-\measuredangle o_{i-1} p o^{\prime} / 2=\measuredangle o_{i-1} p o / 2-\measuredangle o p o^{\prime} / 2
$$


and

It follows that

$$
\measuredangle o_{i-1} p o \geq \measuredangle o_{i-1} p o_{i}
$$

\section{It follows that}

and similarly

$$
\measuredangle v_{i} o_{i} v_{j}^{\prime \prime} \geq \measuredangle v_{i}^{\prime \prime} o v_{j}
$$

$$
\measuredangle v_{i} o_{i} v_{j}^{\prime \prime} \geq \measuredangle v_{i}^{\prime \prime} o v_{j} .
$$

¿From here the proof of the inequality

$$
\varrho_{r}\left(v_{i}^{\prime}, v_{i}\right)+\varrho_{r}\left(v_{i}, v_{j}^{\prime}\right) \geq \varrho_{r}\left(v_{i}^{\prime}, v_{j}\right)+\varrho_{r}\left(v_{j}, v_{j}^{\prime}\right)
$$

follows in the same way as for the Euclidean plane.

Proof of the case of area in Lemma 2 on the sphere and on the hyperbolic plane. The main tool in the Euclidean argument is the technical statement $(*)$. We will show how direct analogues of $(*)$ can be proved on the sphere and on the hyperbolic plane.

The locus of the points $w^{\prime}$ for which the triangles $u v w$ and $u v w^{\prime}$ have the same orientation and area $\left(u v w^{\prime}\right)=$ area $(u v w)$ is a constant distance curve for the line $M$ through $w$, that is, a hypercycle on the hyperbolic plane and the so called Lexell circle on the sphere. Thus the role of the lines $L_{1}$ an $L_{2}$ are played by hypercycles and small circles, respectively. We introduce coordinates so that the origin is the intersection of $M$ and the perpendicular bisector of the segment $u v$, the $y$ coordinate of a point $p$ is the signed distance from $p$ to the orthogonal projection $p_{M}$ of $p$ on $M$, and the $x$ coordinate of $p$ is the signed distance from $p_{M}$ to the origin. Let $\alpha$ denote the central angle of a circular segment of radius $r$ cut off by a chord of length $l$. Then we have

$$
s(l)=\left\{\begin{array}{l}
\pi-\alpha \cos r-2 \operatorname{arccot}\left(\frac{\cos r}{\cot \frac{\alpha}{2}}\right), \alpha=2 \arcsin \left(\frac{\sin \frac{l}{2}}{\sin r}\right) \\
\text { on the sphere, } \\
\alpha \cosh r+2 \operatorname{arccot}\left(\frac{\cosh r}{\cot \frac{\alpha}{2}}\right)-\pi, \alpha=2 \arcsin \left(\frac{\sinh \frac{l}{2}}{\sinh r}\right) \\
\text { on the hyperbolic plane, }
\end{array}\right.
$$

and

$$
l(z)= \begin{cases}2 \arccos \left(\cos \frac{z}{2} \cos y_{0}\right) & \text { on the sphere, } \\ 2 \operatorname{arcosh}\left(\cosh \frac{z}{2} \cosh y_{0}\right) & \text { on the hyperbolic plane. }\end{cases}
$$

It can be checked by direct calculation, which is straightforward but tedious, that both $s(l)$ and $l(z)$ are strictly monotonically increasing and convex in both geometries. The anonymous referee suggested the following alternate argument to verify the monotonicity and convexity of $s(l)$ and $l(z)$.

The function $l(z)$ is twice the length of the hypotenuse of a right triangle whose catheti have lengths $y_{0}$ and $z / 2$, and where $y_{0}$ is fixed. If we draw two such right triangles in a way that they share the right angle and the leg of length $y_{0}$, cf. Figure 4, then the monotonicity of $l$ is clear from the fact that in a triangle the larger side is opposite to the larger angle. Convexity of $l$ is a consequence of the theorem that the length of a median of a triangle is less than the mean of the sides sharing a vertex with it. (This follows easily from the triangle inequality applying a central reflection in the midpoint of the third side of the triangle.)

The convexity of $s(l)$ can be proved as follows. If $K= \pm 1$ is the sectional curvature of the plane, then the sides $a, b, c$ of a right triangle, where $c(\leq \pi / 2$ in 


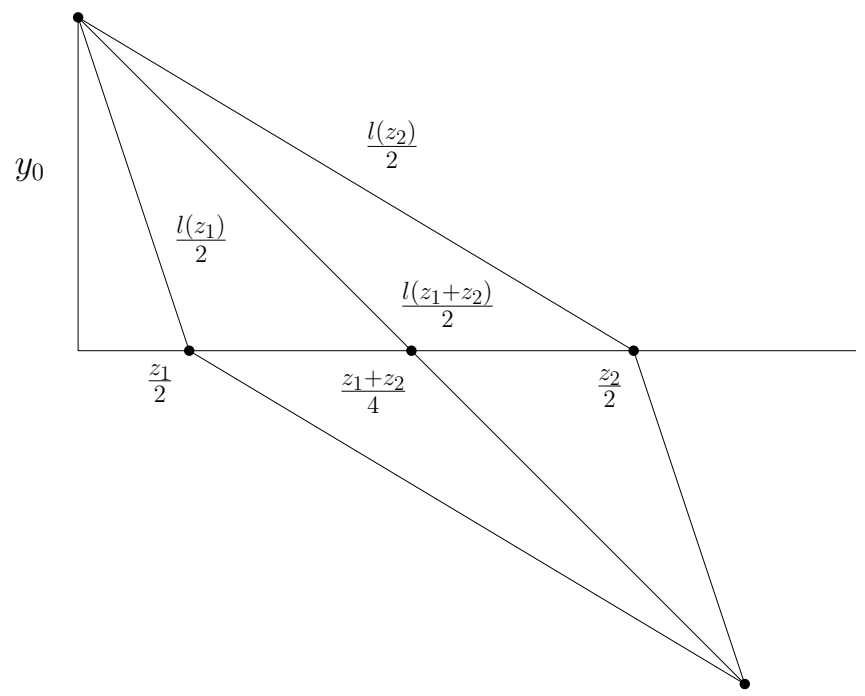

Figure 4. The function $l(z)$.

the spherical case) is the hypothenuse, are related to one another by the formula

$$
\cos (\sqrt{K} a) \cos (\sqrt{K} b)=\cos (\sqrt{K} c) .
$$

If we fix the hypotenuse $c$ and express $b$ as a function $b=f_{c}(a)$ of $a \in[0, c]$, then by differentiating the equation

$$
\cos (\sqrt{K} a) \cos \left(\sqrt{K} f_{c}(a)\right)=\cos (\sqrt{K} c)
$$

with respect to $a$, we obtain that

$$
f_{c}^{\prime}(a)=-\frac{\tan (\sqrt{K} a)}{\tan \left(\sqrt{K} f_{c}(a)\right)} .
$$

This yields that $f_{c}^{\prime}(a)$ is negative for all $a \in[0, c]$, and hence, $f_{c}$ is strictly decreasing on $[0, c]$. By differentiating (3) one more time with respect to $a$ and using the monotonicity of the tangent and hyperbolic tangent functions, we obtain that $f_{c}^{\prime}$ is also strictly monotonically decreasing on $[0, c]$, which yields that $f_{c}$ is concave.

Denote by $p(r)=2 \pi \frac{\sin (\sqrt{K} r)}{\sqrt{K}}$ the perimeter of a circle of radius $r$ and by $a_{r}(l)$ the length of the shorter arc of this circle cut off by a chord of length $l$. Consider a circle of perimeter $p(r)$ in the Euclidean plane and denote by $W_{r}(l)$ the length of the chord of this circle that cuts off an arc of length $a_{r}(l)$.

The area $s(l)$ is half the area of the intersection of two discs of radius $r$ that share a chord of length $l$. Thus, using Theorem 5.1 of Csikós [7], we obtain that

$$
s^{\prime}(l)=-f_{r}^{\prime}(l / 2) W_{r}(l) .
$$

Since $W_{r}$ is positive and strictly increasing and $f_{r}^{\prime}$ is negative and decreasing, it follows that $s^{\prime}$ is positive and increasing, which, in turn, yields that $s$ is increasing and convex.

Now we can finish the proof in the same way as in the Euclidean case with the only difference that the role of the line $L$ is played by the constant distance curve for the line $v_{i-1} v_{j-1}$ through $v_{j}$. 


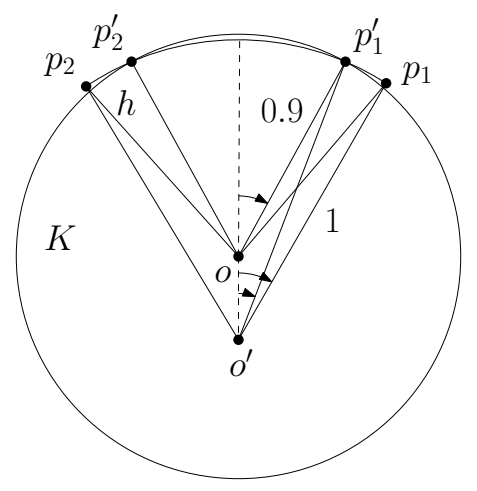

Figure 5 .

For two convex discs $K_{1}$ and $K_{2}$ let their area deviation $\delta_{a}\left(K_{1}, K_{2}\right)$ and perimeter deviation $\delta_{p}\left(K_{1}, K_{2}\right)$ be defined as

$$
\delta_{a}\left(K_{1}, K_{2}\right)=\operatorname{area}\left(K_{1} \cup K_{2}\right)-\operatorname{area}\left(K_{1} \cap K_{2}\right)
$$

and

$$
\delta_{p}\left(K_{1}, K_{2}\right)=\operatorname{per}\left(K_{1} \cup K_{2}\right)-\operatorname{per}\left(K_{1} \cap K_{2}\right) .
$$

For a convex disc $K$ let $d_{a}(n)$ and $d_{p}(n)$ denote the minimum area deviation and the minimum perimeter deviation of an $n$-gon from $K$, respectively. Eggleston [10] proved that both $d_{a}(n)$ and $d_{p}(n)$ are convex functions of $n$. It is an interesting question whether the minimum area deviation and the minimum perimeter deviation of a disc- $n$-gon of radius $r$ from a hyperconvex disc $K$ are also convex functions of $n$. To answer this question seems to be difficult. As one source of the difficulty in the case of the perimeter deviation we mention the following. Eggleston [10] proved that for a convex disc $K$ among all convex $n$-gons the one closest to $K$ in the sense of perimeter deviation is always inscribed in $K$. The following example shows that if $K$ is a hyperconvex disc, then its best approximating disc- $n$-gon in the sense of perimeter deviation may neither be inscribed in nor circumscribed about $K$. Let $K$ be the closed circular discs of radius 0.9 centred at the origin $o$. Let $p_{1}$ be an arbitrary point with $d\left(p_{1}, o\right)=h$ for some arbitrary fixed $0<h<1$. Then there exists a unique regular disc-pentagon $P_{5}(h)$ of radius 1 centred at $o$ with one of its vertices equal to $p_{1}$. Note that the disc-pentagon $P_{5}(0.9)$ is inscribed in $K$. Let $h_{1}$ be the value of $h$ for which $P_{5}\left(h_{1}\right)$ is circumscribed about $K$.

Theorem 2 implies, on one hand, that $p_{i}(5)=\operatorname{per}\left(P_{5}(0.9)\right)=5.565 \ldots$, on the other hand, that $p_{c}(5)=\operatorname{per}\left(P_{5}\left(h_{1}\right)\right)=5.690 \ldots$ Hence $\delta_{p}\left(P_{5}(0.9), K\right)=$ $1.8 \pi-5.565 \ldots=0.080 \ldots, \delta_{p}\left(P_{5}\left(h_{1}\right), K\right)=5.690 \ldots-1.8 \pi=0.04 \ldots$. Thus, $P_{5}\left(h_{1}\right)$ is closer to $K$ than $P_{5}(0.9)$ in the sense of perimeter deviation. Now, we will examine the case when $h \in\left(0.9, h_{1}\right)$. Let the vertices of $P_{5}(h)$ be labeled in the positive direction and let $p_{1}^{\prime}$ and $p_{2}^{\prime}$ be the intersection points of the unit radius $\operatorname{arc} p_{1} p_{2}$ and bd $K$ as shown on Figure 5. Let $\alpha=\measuredangle o o^{\prime} p_{1}, \beta=\measuredangle o o^{\prime} p_{1}^{\prime}$, and $\gamma=\pi-\measuredangle p_{1}^{\prime} o o^{\prime}$, cf. Figure 5. It is clear that

$$
\begin{aligned}
\delta_{p}\left(P_{5}(h), K\right) & =\operatorname{per}\left(P_{5}(h) \cup K\right)-\operatorname{per}\left(P_{5}(h) \cap K\right) \\
& =(10(\alpha-\beta)+9 \cdot \gamma)-(10 \beta+(2 \pi-10 \gamma) \cdot 0.9) .
\end{aligned}
$$




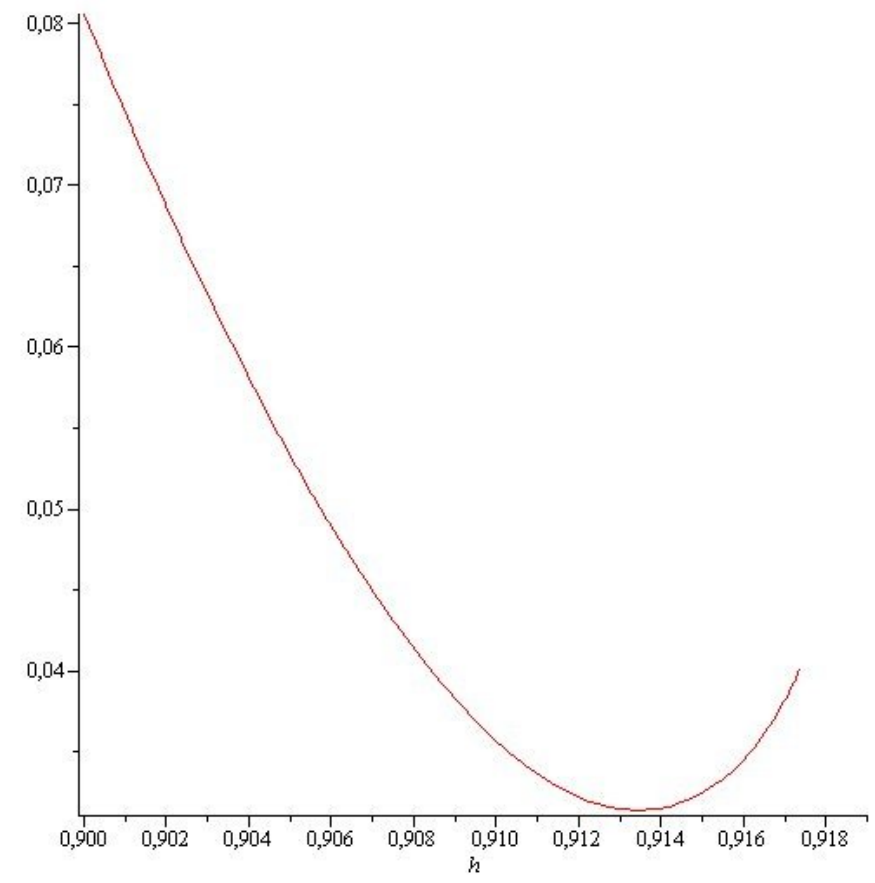

Figure 6 . The graph was drawn by Maple 13.0

The right hand side of (4) can be expressed explicitly in terms of $h$ using basic trigonometry; we leave the detailed calculations to the reader. The graph of (4) is shown on Figure 6.

It is apparent from Figure 6 that there is a whole subinterval of positive length of $\left(0.9, h_{1}\right)$ in which $P_{5}(h)$ approximates $K$ better than both $P_{5}(0.9)$ and $P_{5}\left(h_{1}\right)$ in the sense of perimeter deviation. Thus the best approximating disc-pentagon of unit radius of $K$ is neither inscribed in nor circumscribed about $K$.

\section{ACKnowledgements}

The authors thank the anonymous referee whose suggestions have greatly improved the article. In particular, the arguments that show the monotonicity and convexity of the functions $s(l)$ and $l(z)$ were suggested by the referee.

The first author was supported by Hungarian OTKA grant K76154 and by the Fields Institute for Research in Mathematical Sciences.

The second author was supported by the János Bolyai Research Scholarship of the Hungarian Academy of Sciences, Hungarian OTKA grant K75016, and by the Fields Institute for Research in Mathematical Sciences.

\section{REFERENCES}

[1] K. Bezdek, Lectures on sphere arrangements - the discrete geometric side, Fields Institute Monographs, vol. 32, Springer, New York, 2013.

[2] K. Bezdek, Classical topics in discrete geometry, CMS Books in Mathematics/Ouvrages de Mathématiques de la SMC, Springer, New York, 2010. 
[3] K. Bezdek, R. Connelly, and B. Csikós, On the perimeter of the intersection of congruent disks, Beiträge Algebra Geom. 47 (2006), no. 1, 53-62.

[4] K. Bezdek, Z. Lángi, M. Naszódi, and P. Papez, Ball-polyhedra, Discrete Comput. Geom. 38 (2007), 201-230.

[5] E. Blanc, Les ensembles surconvexes plans, Ann. Sci. École Norm. Sup. (3) 60 (1943), 215246.

[6] J. Buter, Überconvexe Mengen in der Ebene, Ak. Wetensch. Amsterdam Proc. 41 (1938).

[7] B. Csikós, On the volume of flowers in space forms, Geom. Dedicata 86 (2001), 59-79.

[8] L. Danzer, B. Grünbaum, and V. Klee, Helly's theorem and its relatives, Proc. Sympos. Pure Math., Vol. VII, Amer. Math. Soc., Providence, R.I., 1963, pp. 101-180.

[9] C. H. Dowker, On minimum circumscribed polygons, Bull. Amer. Math. Soc. 50 (1944), 120-122.

[10] H. G. Eggleston, Approximation to plane convex curves. (I) Dowker-type theorems, Proc. London Math. Soc. (3) 7 (1957), 351-377.

[11] G. Fejes Tóth and L. Fejes Tóth, Remark on a paper of C. H. Dowker, Period. Math. Hungar. 3 (1973), 271-274.

[12] L. Fejes Tóth, Some packing and covering theorems, Acta Sci. Math. Szeged 12 (1950), no. Leopoldo Fejér et Frederico Riesz LXX annos natis dedicatus, Pars A, 62-67.

[13] L. Fejes Tóth, Remarks on polygon theorems of Dowker, Mat. Lapok 6 (1955), 176-179 (Hungarian).

[14] L. Fejes Tóth, Annäherung von Eibereichen durch Polygone, Math.-Phys. Semesterber. 6 (1958/1959), 253-261.

[15] L. Fejes Tóth, Packing of $r$-convex discs, Studia Sci. Math. Hungar. 17 (1982), 449-452.

[16] L. Fejes Tóth, Packing and covering with r-convex discs, Studia Sci. Math. Hungar. 18 (1982), 69-73.

[17] Y. S. Kupitz, H. Martini, and M. A. Perles, Finite sets in $\mathbb{R}^{d}$ with many diameters - a survey, Proceedings of the International Conference on Mathematics and Applications (ICMA-MU 2005, Bangkok), Mahidol University Press, Bangkok, 2005, pp. 91-112. Reprinted in a special volume of the East-West J. Math.: Contributions in Mathematics and Applications (2007), $41-57$.

[18] Y. S. Kupitz, H. Martini, and M. A. Perles, Ball polytopes and the Vázsonyi problem, Acta Math. Hungar. 126 (2010), 99-163.

[19] A. E. Mayer, Eine Überkonvexität, Math. Z. 39 (1935), 511-531.

[20] J. Molnár, On inscribed and circumscribed polygons of convex regions, Mat. Lapok 6 (1955), 210-218 (Hungarian).

[21] L. Pasqualini, Superconvexité, Bull. Cl. Sci. Acad. Belg. (5) 25 (1939), 18-24.

[22] L. A. Santaló, On plane hyperconvex figures, Summa Brasil. Math. 1 (1946), 221-239 (Spanish).

[23] J. G. van der Corput, Überkonvexe Mengen in der Ebene, Ak. Wetensch. Amsterdam Proc. 41 (1938).

[24] P. Vincensini, Sur les figures superconvexes planes, Bull. Soc. Math. France 64 (1936), 197208.

Alfréd Rényi Institute of Mathematics Hungarian Academy of Sciences, Reáltanoda u. 13-15., H-1053, Budapest, Hungary

E-mail address: gfejes@renyi.hu

Department of Geometry, Bolyai Institute, University of Szeged, Aradi vértanúk tere 1, H-6720 Szeged, Hungary and Department of Mathematics and Statistics, University of Calgary, 2500 University Dr. N.W., Calgary, Alberta, Canada, T2N 1N4

E-mail address: fodorf@math.u-szeged.hu 